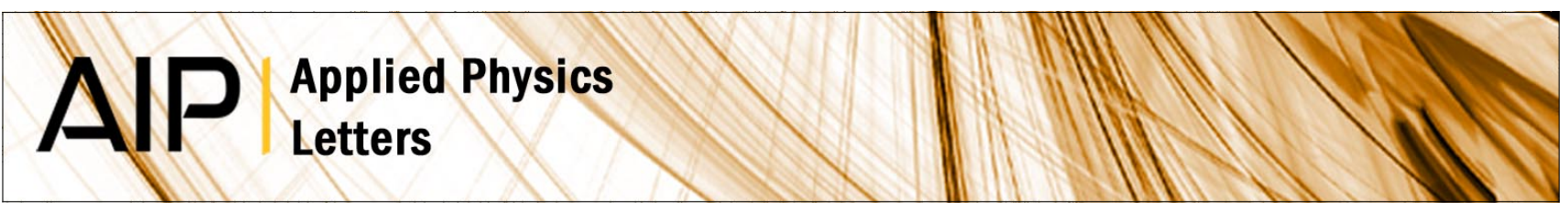

\title{
Rapid, substrate-independent thickness determination of large area graphene layers
}

Dinesh K. Venkatachalam, Patrick Parkinson, Simon Ruffell, and Robert G. Elliman

Citation: Appl. Phys. Lett. 99, 234106 (2011); doi: 10.1063/1.3664633

View online: http://dx.doi.org/10.1063/1.3664633

View Table of Contents: http://apl.aip.org/resource/1/APPLAB/v99/i23

Published by the American Institute of Physics.

\section{Related Articles}

Quantum Hall effect on centimeter scale chemical vapor deposited graphene films Appl. Phys. Lett. 99, 232110 (2011)

A transfer technique for high mobility graphene devices on commercially available hexagonal boron nitride Appl. Phys. Lett. 99, 232104 (2011)

Low-temperature graphene growth using epochal catalyst of PdCo alloy

Appl. Phys. Lett. 99, 223102 (2011)

Synchrotron x-ray analyses of crystalline and electronic structures of carbon nanowalls Appl. Phys. Lett. 99, 213110 (2011)

Thermal rectification in thickness-asymmetric graphene nanoribbons

Appl. Phys. Lett. 99, 193104 (2011)

\section{Additional information on Appl. Phys. Lett.}

Journal Homepage: http://apl.aip.org/

Journal Information: http://apl.aip.org/about/about_the_journal

Top downloads: http://apl.aip.org/features/most_downloaded

Information for Authors: http://apl.aip.org/authors

\section{ADVERTISEMENT}

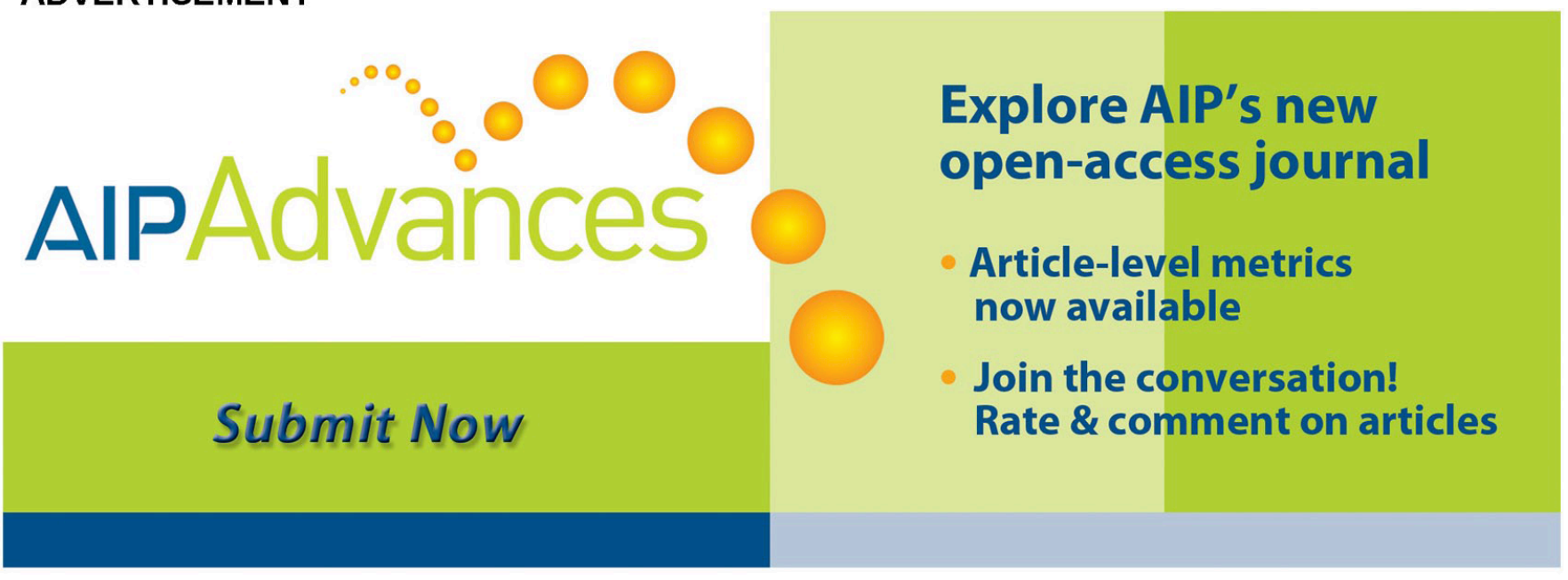




\title{
Rapid, substrate-independent thickness determination of large area graphene layers
}

\author{
Dinesh K. Venkatachalam, ${ }^{\text {a) }}$ Patrick Parkinson, Simon Ruffell, and Robert G. Elliman \\ Department of Electronic Materials Engineering, Research School of Physics and Engineering, \\ Australian National University, Canberra ACT 0200, Australia
}

(Received 15 September 2011; accepted 8 November 2011; published online 9 December 2011)

\begin{abstract}
Phase-shifting interferometric imaging is shown to be a powerful analytical tool for studying graphene films, providing quantitative analysis of large area samples with an optical thickness resolution of $\leq 0.05 \mathrm{~nm}$. The technique is readily able to identify single sheets of graphene and to quantitatively distinguish between layers composed of multiple graphene sheets. The thickness resolution of the technique is shown to result from the phase shift produced by a graphene film as incident and reflected light pass through it, rather than from path-length differences produced by surface height variations. This is enhanced by the high refractive index of graphene, estimated in this work to be $\mathrm{n}_{\mathrm{G}}=2.99 \pm 0.18$. (C) 2011 American Institute of Physics. [doi:10.1063/1.3664633]
\end{abstract}

There is considerable fundamental ${ }^{1,2}$ and technologi$\mathrm{cal}^{2,3}$ interest in the synthesis, properties, and applications of graphene. However, from a practical point-of-view, it can be quite difficult to locate and identify single layers of graphene on a substrate due to their small physical thickness and optical transparency. Optical detection is especially attractive because it offers the potential for rapid, nondestructive characterization of large-area samples. This can be achieved by conventional optical microscopy but requires that the graphene layers be transferred to an engineered substrate, typically a thin film structure, to increase their optical contrast. ${ }^{4-6}$ Recently, a similar method has been used for the identification of single graphene sheets deposited on a $\mathrm{Cu}$ substrate. ${ }^{7}$ Because this technique relies on small contrast differences to distinguish layers of different thickness, it is often subject to ambiguity, particularly when graphene is only a minor component of the film. As a consequence, highly sophisticated image processing tools are required to image graphene directly on metallic substrates such as $\mathrm{Ni}$ or $\mathrm{Cu}$ that are commonly used for large area synthesis of graphene by chemical vapour deposition ${ }^{8}$ or by ion implantation $^{9}$ techniques. Ellipsometry has recently emerged as a viable alternative technique but also requires properly designed substrates. ${ }^{10}$ Thus, rapid measurement of graphene on different substrates over large areas but with high lateral resolution is extremely desirable. In addition, the approach needs to be robust so that measurement setup and artifacts do not affect the results.

In this study, we introduce phase-shifting interferometric imaging as a simple and fast optical detection method for the rapid identification and characterization of graphene layers. In principal, this technique can be used to characterize graphene layers on a variety of substrates, and of an arbitrary number of layers, and offers the potential for in-situ observation of physical changes induced by graphene processing, such as oxidation.

Samples consisting of graphene flakes of different thickness on $\mathrm{a} \mathrm{SiO}_{2}(300 \mathrm{~nm}) / \mathrm{Si}$ substrate were purchased from

\footnotetext{
a) Author to whom correspondence should be addressed. Electronic mail: Dinesh.Venkatachalam@anu.edu.au.
}

Graphene Industries Limited (Manchester, UK). The flakes were deposited using the exfoliation technique. ${ }^{11}$ As a part of the present study, samples were characterized by conventional optical microscopy and Raman spectroscopy in addition to the phase-shifting interferometry (PSI). Optical imaging was undertaken with an Olympus BX41 microscope fitted with a $100 \times$ objective lens using normal-incidence, white light illumination. PSI images were measured using a Veeco (Wyko NT9100) optical profiler operating with a source wavelength defined by a $10 \mathrm{~nm}$ band-pass filter centered at a wavelength of $525 \mathrm{~nm}$.

The optical contrast of graphene layers can be enhanced by thin-film interference effects, which in the present case is achieved by using a $300 \mathrm{~nm} \mathrm{SiO}_{2} / \mathrm{Si}$ substrate for the samples. A typical optical image of the supplied graphene sample is, shown in Fig. 1(a), clearly showing light and dark regions that can be correlated with 1,2 , or $3+$ graphene layers, based on the optical density ${ }^{12}$ and sample-maps provided by the supplier. The assignment of the different step heights was independently confirmed by Raman spectroscopy (results not shown).

For comparison, Fig. 1(b) shows a phase-shifting image of the same region collected with the optical profiler in less than $60 \mathrm{~s}$. This clearly identifies regions with four different step heights corresponding to the substrate $\left(\mathrm{SiO}_{2}\right)$ and mono-, bi-, and tri- layer graphene sheets, as labeled. Although not immediately evident from Fig. 1(b), the measured step heights of the different graphene layers are inverted relative to their expected physical thickness, with the $\mathrm{SiO}_{2}$ substrate appearing higher than regions covered with graphene, and thicker graphene layers appearing lower than thinner ones. This is more clearly seen in Fig. 1(c) which shows a threedimensional view of a selected area of the sample [marked by a rectangle in Fig. 1(b)]. To understand this inverted topology, it is necessary to consider the phase-shifting measurement in more detail. To construct a topological map from PSI data, the relative step height between neighboring regions on a surface is calculated from the measured phase difference as follows:

$$
\Delta h_{12}=\frac{\lambda}{4 \pi} \Delta \phi_{12}
$$


where $\lambda$ is the wavelength of the source beam and $\Delta \phi_{12}$ is the position-dependent phase-difference data. For light incident normal to a reflective surface, this is straight forward, and a positive phase difference between region 1 and region 2, $\Delta \phi_{12}=\phi_{1}-\phi_{2}>0$, indicates that region 1 is higher than region 2 , i.e., the reflection path length is longer for the lower region than from the higher region. Under the phaseshift interferometry technique, the strongest reflection is used as the "sample arm" of the interferometer, which is a function of the refractive index, thickness of each layer, and the wavelength used to probe the sample. However, the reflectivity of a layer of graphene on silica is extremely low (less than 4\%), and a simple calculation for the present sample structure ${ }^{13}$ shows that the strongest reflection comes from the silica-silicon interface. By comparing the optical path lengths of the most strongly reflected beam with and without a graphene layer, it can be seen that the former introduces a phase shift equal to two passes through the graphene layer, such that

$$
\Delta \phi=+\frac{4 \pi}{\lambda}\left(t_{G} n_{G} \pm \delta\right),
$$

where $t_{G}$ and $n_{G}$ are thickness and refractive index of the graphene layer and $\delta$ is an optical path contribution from adsorbates or residues that are specific to the substrate or graphene layers. The phase-shift defined by Eq. (2) manifests itself as an increased sample arm length, and is therefore interpreted as coming from a "deeper" layer or a "recess" relative to the silica surface as observed in Fig. 1(c). Importantly, in cases where the reflection from the graphene surface is stronger than that from the substrate, the sign of the apparent thickness will swap and Eq. (2) becomes

$$
\Delta \phi=-\frac{4 \pi}{\lambda}\left(t_{G} n_{A} \pm \delta\right)
$$

(a)

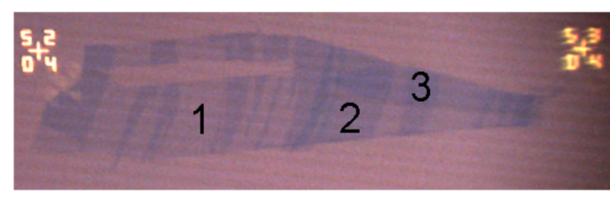

(b)

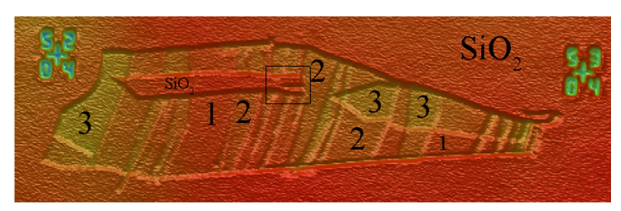

(c)

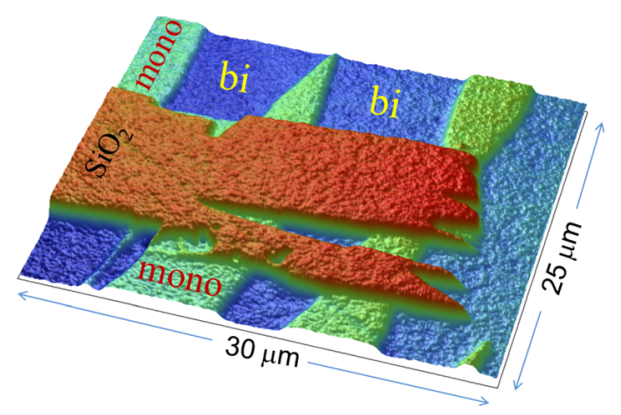

FIG. 1. (Color online) (a) Optical image of graphene film on a $\mathrm{SiO}_{2} / \mathrm{Si}$ substrate measured under white light illumination. NB: The distance between markers is $0.4 \mathrm{~mm}$, (b) a PSI image of the graphene sample depicted in (a), and (c) a 3D PSI image of a region of the graphene sample, as indicated by the square in (b). where $n_{A}$ is the refractive index of the imaging ambient (air in the current experiments), and the graphene layer will appear as a positive step on the substrate. Significantly, the measured phase shift does not depend on details of the substrate structure. All that is required for high-quality imaging is reflection from some interface, and a sufficiently low surface roughness such that the edges of the graphene flake are visible.

A particular strength of this technique is that it can provide rapid quantitative analysis of over large area samples, with typical images taking just a few seconds. As an example, Fig. 2(a) presents a PSI image of a $140 \mu \mathrm{m} \times 75 \mu \mathrm{m}$ region of the sample, together with line-scans showing "apparent" step height profiles across particular paths, Fig. 2(b). The step height is initially calculated assuming a refractive index of 1.0 and as a consequence indicates the optical thickness of the layers.

To calibrate the measured data, the optical thicknesses of regions containing one, two, and three layers of graphene were determined by direct measurements between the substrate and the layers, as shown in Fig. 3(a). As expected, the measured average optical thicknesses show a linear dependence with increase in the number of graphene layers, with a slope, $t_{G} n_{G}$, corresponding to $2.0 \pm 0.12 \mathrm{~nm}$ per layer and an offset, $\delta$, of $0.6 \pm 0.26 \mathrm{~nm}$. Using a typical value for the physical thickness of graphene of $0.335 \mathrm{~nm},{ }^{11}$ this corresponds to a refractive index of $2.99 \pm 0.18$-more than that associated with bulk graphite ${ }^{5}$ and within the range reported by others. ${ }^{14}$ Figure 3 (b) shows a PSI image of a selected region of the sample, together with line scans calibrated using this data [Fig. 3(c)]. The step heights between the substrate and two-layers of graphene and between two- and

(a)

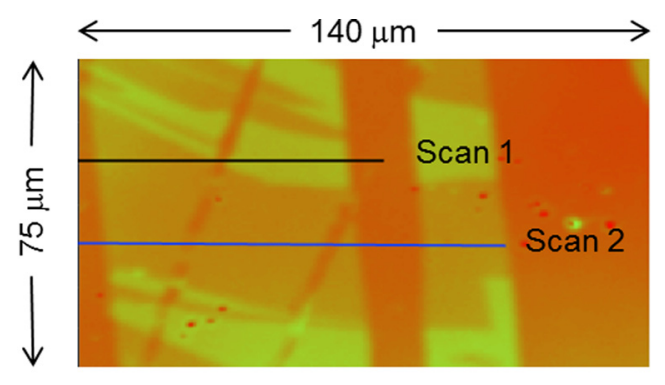

(b)

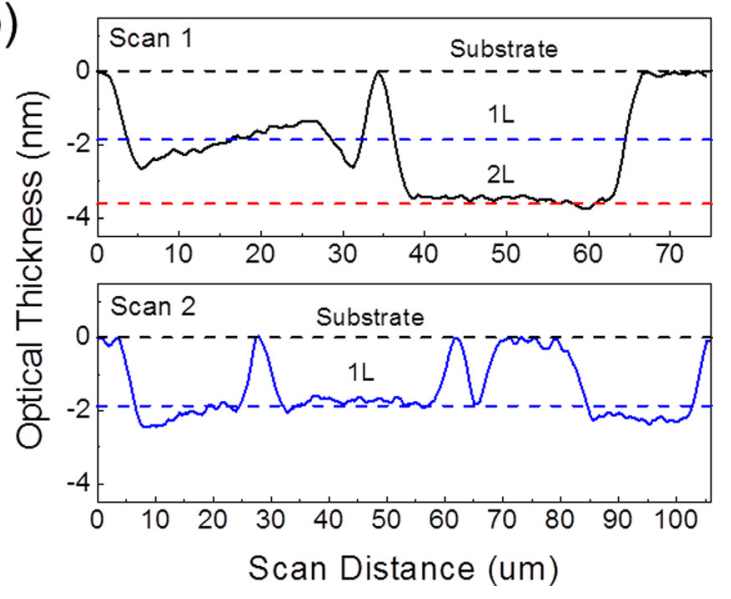

FIG. 2. (Color online) (a) PSI image of a selected region of the graphene sample with lines indicating the trajectory of line scans 1 and 2, and (b) a plot of optical thickness versus position for scans 1 and 2 . 
(a)

(b)
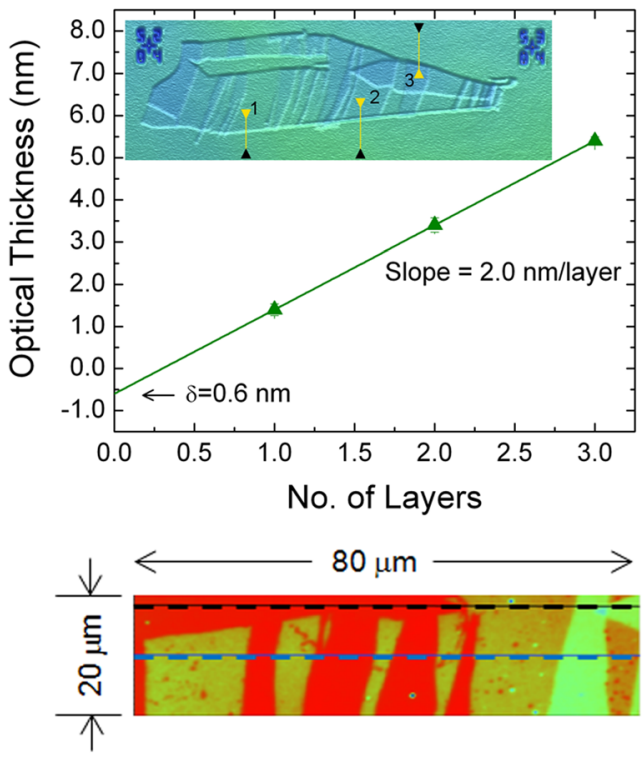

(c)

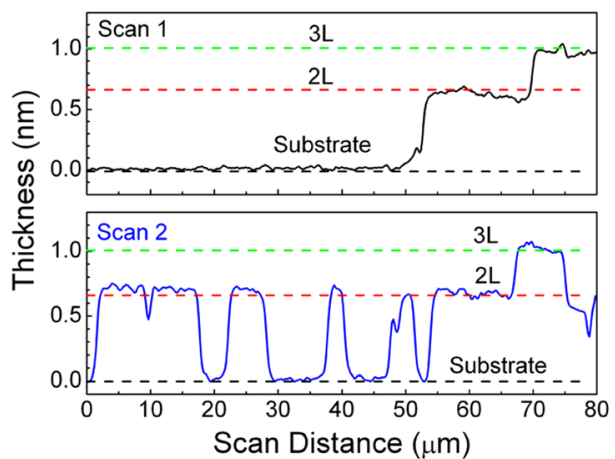

FIG. 3. (Color online) (a) Plot of measured average optical thickness as a function of the number of graphene layers. Inset shows a PSI image of the graphene sample showing the position of line scans used for the thickness measurements, (b) PSI image of a selected region of the graphene sample with lines indicating the trajectory of line scans 1 and 2, and (c) calibrated line scan showing the graphene film thickness along line scans 1 and 2 .

three-layers of graphene are clearly evident, and the signalto-noise ratio is consistent with a resolution of $\leq 0.05 \mathrm{~nm}$.

Commercially available optical profilers, such as that employed in the present work, are capable of measuring surface height variations of $<0.1 \mathrm{~nm}$ in air, which corresponds to $<0.03 \mathrm{~nm}$ thickness variations in graphene, consistent with the results presented in Fig. 3(c), and sufficient to observe graphene-graphene oxide transitions. The technique is also capable of imaging large surface areas, with field of view and lateral spatial resolution ranging from $\leq 50 \mu \mathrm{m}$ to
$>2 \mathrm{~mm}$ and $\leq 160 \mathrm{~nm}$ to $>7 \mu \mathrm{m}$, respectively, depending on the choice of camera resolution and lenses. Although not optimized for high spatial resolution, scan 2 in Fig. 3 clearly shows the presence of a pin hole, located at a scan distance of $10 \mu \mathrm{m}$. The power of the technique is further enhanced by its ability to undertake in-situ investigations of samples during thermal or other processing.

In summary, phase-shifting interferometric imaging was shown to provide quantitative thickness analysis of largearea graphene films and to be readily able to distinguish films composed of single or multiple graphene sheets. The sensitivity of the technique was shown to result from the phase shift produced by the graphene film as incident and reflected light pass through it, rather than from path-length differences produced by surface height variations, as is usually the case for PSI imaging. This was enhanced by the high refractive index of graphene, estimated in the present work to be $\mathrm{n}_{\mathrm{G}}=2.99 \pm 0.18$. Importantly, this technique has the potential to characterize films consisting of an arbitrary number of graphene sheets on a variety of substrates and offers the sensitivity for in-situ observation of physical changes induced by graphene processing, such as oxidation.

The authors wish to acknowledge Barry Luther-Davies for useful discussions and assistance with optical-profiling measurements and the Australian Research Council for financial support.

${ }^{1}$ A. K. Geim and K. S. Novoselov, Nature Mater. 6(3), 183 (2007).

${ }^{2}$ Y. H. Wu, T. Yu, and Z. X. Shen, J. Appl. Phys. 108(7), 071301 (2010).

${ }^{3}$ M. D. Stoller, S. Park, Y. Zhu, J. An, and R. S. Ruoff, Nano Lett. 8(10), 3498 (2008)

${ }^{4}$ D. S. L. Abergel, A. Russell, and I. F. Vladimir, Appl. Phys. Lett. 91(6), 063125 (2007).

${ }^{5}$ P. Blake, E. W. Hill, A. H. C. Neto, K. S. Novoselov, D. Jiang, R. Yang, T. J. Booth, and A. K. Geim, Appl. Phys. Lett. 91(6), 063124 (2007).

${ }^{6}$ S. Roddaro, P. Pingue, V. Piazza, V. Pellegrini, and F. Beltram, Nano Lett. 7(9), 2707 (2007).

${ }^{7}$ Q. Yu, L. A. Jauregui, W. Wu, R. Colby, J. Tian, Z. Su, H. Cao, Z. Liu, D. Pandey, D. C. Wei et al., Nature Mater. 10(6), 443 (2011).

${ }^{8}$ A. Reina, X. Jia, J. Ho, D. Nezich, H. Son, V. Bulovic, M. S. Dresselhaus, and J. Kong, Nano Lett. 9(1), 30 (2008).

${ }^{9}$ S. Garaj, W. Hubbard, and J. A. Golovchenko, Appl. Phys. Lett. 97(18), 183103 (2010).

${ }^{10}$ W. Ulrich, R. Christian, W. Ursula, W. Werner, V. Matthias, H. T. Peter, and W. Dieter, Appl. Phys. Lett. 97(23), 231901 (2010).

${ }^{11}$ K. S. Novoselov, A. K. Geim, S. V. Morozov, D. Jiang, Y. Zhang, S. V. Dubonos, I. V. Grigorieva, and A. A. Firsov, Science 306(5696), 666 (2004).

${ }^{12}$ Y.-F. Chen, D. Liu, Z.-G. Wang, P.-J. Li, X. Hao, K. Cheng, Y. Fu, L.-X. Huang, X.-Z. Liu, W.-L. Zhang et al., J. Phys. Chem. C 115(14), 6690 (2011).

${ }^{13}$ H. Anders, Thin Films in Optics (Focal, London, 1967).

${ }^{14}$ M. Bruna and S. Borini, Appl. Phys. Lett. 94(3), 031901 (2009). 